\title{
Grid-aware Distributed Control of Electric Vehicle Charging Stations in Active Distribution Grids
}

\author{
Sherif Fahmy, Rahul Gupta, Mario Paolone \\ Distributed Electrical Systems Laboratory \\ École Polytechnique Fédérale de Lausanne, DESL-EPFL \\ Lausanne, Switzerland \\ \{sherif.fahmy, rahul.gupta, mario.paolone\}@epfl.ch
}

\begin{abstract}
The penetration of electric vehicle (EV) charging stations (CSs), along with the progressive connection of stochastic distributed generation, is increasing the probability of violating the power distribution grid operational constraints and deteriorate the quality of power supply. To this end, the paper proposes a real-time control scheme for allocating power set-points to EV CSs while accounting for the grid operational requirements. In the proposed problem formulation the grid and the power injections are modelled accounting for their unbalanced 3-phase nature, thus enabling to formulate the problem objective and its constraints adopting the sequence decomposition. The EVs' users need, along with the stochastic nature of other uncontrollable injections (e.g. loads and generation from photovoltaic generation units), are also taken into account. A distributed control scheme, with a minute-scale control horizon, is proposed where local controllers, operating at EV aggregation level, compute EV batterysecure power set-points. These controllers send their set-points to a central controller operating at the grid aggregation level. The central controller solves a scenario-based linearised optimal power flow accounting for grid operational and power quality constraints. Then, it sends back its solution to the respective local controllers. The obtained iterative algorithm is efficiently solved until convergence. We analyse the performance of the proposed control scheme via a set of simulation ran on the IEEE-34 feeder. Comparisons with two other control algorithms, a grid-unaware local controller and a myopic maximum power controller, are included to benchmark the proposed control scheme.
\end{abstract}

Index Terms-Electric Vehicles Charging Stations, Real-Time Distributed Control, Grid Security and Power Quality.

\section{INTRODUCTION}

The electrification of the private transportation sector, along with a move towards distributed renewable generation, are being proposed as early solutions to reduce $\mathrm{CO}_{2}$ emissions. Due to their high-power demand, the deployment of electric vehicle (EV) charging stations (CSs) is increasing the probability of violating static distribution grid operational constraints (i.e., exceeding branch ampacity limits, nodal under/over-voltages and substation transformers overloads) [1]. Furthermore, with the increased penetration of single-phase EV chargers, the risk of voltage unbalances is high, thus, deteriorating the power quality of distribution grids. The complexity behind developing control schemes that deal with the latter point originates from several factors: the stochastic behaviour of EV users, the high EV power demand, the stochasticity of renewable distributed generation that might not coincide with EV peak load demands and the scalability of centralized control charging schemes. In this regard, the paper proposes a real-time (i.e. seconds to minutes scale) distributed EV power allocation algorithm that takes into account EV users' needs, grids' static and power quality constraints, and the stochastic nature of power generation units and loads.

Centralised and distributed real-time algorithms for EV scheduling and optimal charging that account for grid constraints can be found in the literature. In, [2], a distributed locally optimal scheduling algorithm for EV CSs that determines optimal charging/discharging powers is proposed. The local algorithms take into account EV user needs, battery lifetime and local energy balances using grid forecasts sent by a centralized grid observer. In [3] and [4], two centralised gridaware EV power allocation algorithms are developed. In [3], Authors develop an iterative algorithm that first computes EV power allocations that satisfy user demands based on priority schemes. Then, it verifies through load-flow calculus whether the allocations satisfy the static grid voltage constraints; in case they do not, charging is postponed to the next timestep. In [4], the proposed algorithm assumes that grids are balanced three-phase, linearizes the power-flow equations and accounts for substation transformer maximum power capacity and voltage magnitude violations at nodes where CSs are connected. In [5] and [6], two decentralised grid-aware EV power allocation algorithms are developed. In [5], assuming that the grid is radial and balanced, a lossless LinDistFlow power-flow linearization is used to develop a decentralised EV charging control scheme to achieve EV energy demand while maximizing grid peak-load shaving. In [6], assuming that grids are radial, "roughly balanced" and that losses are "relatively small", a three-phase power-flow linearization using the branch-flow model is used to develop a decentralised EV charging protocol. Both algorithms in [5] and [6] incorporate EV user needs, however, although battery degradation is considered in [5], they both do not consider non-EV load and generation stochasticity. With the exception of [6], all these works assume balanced three-phase grids thus neglecting the impact that single-phase EV charging may have on voltage unbalance.

Unlike all the presented works, we propose a real-time decentralised control scheme to allocate active (charging) and reactive power setpoins to EV CSs. We directly account in the problem for the operational constraints (voltages, branches 
and transformers ampacity limits) and power quality of grids without any assumptions on grid topology model and losses. To do so, we use a three-phase OPF modelled by the powerflow linearisation based on sensitivity coefficients [7]. Furthermore, uncontrollable and stochastic loads and generation are taken into account by leveraging scenario-based optimisation. Additionally, EV user needs (i.e. reaching target state-ofcharges at specified departure times), EV battery wearing and EV charging modes (i.e. three-phase, single-phase and DC charging) are all included in the problem formulation. Finally, to guarantee scalability, the problem is formulated in a separable form enabling the use of the alternating direction method of multipliers (ADMM) to decompose and solve the problem in a distributed manner. ADMM guarantees to converge to the solution of the non-distributed version of the proposed problem as power-flow equations are linearised. As known, the ADMM splits the non-distributed problem into local problems and a central aggregator problem. In the proposed method, local problems satisfying EV user needs are solved at the level of EV aggregators of the nodes where there are EV CSs and do not require any knowledge of the grid parameters, and the central aggregator problem is solved at the level of a distribution grid operator (grid aggregator). Indeed, the grid aggregator coordinates with all EV aggregators to guarantee the satisfaction of grid security constraints and power quality under injection uncertainty. It is therefore assumed that, the developed method is aimed to be used by distribution grid operators to ensure secure EV charging.

In summary, the main contributions of this paper are: i) a formulation of a real-time control scheme to account for grid operational and power quality constraints ii) a formulation of a generalized control scheme addressing the unbalanced nature of $\mathrm{EV}$ chargers, iii) to propose a separable formulation of the problem that decouples the grid and EV CSs aggregators' objectives and constraints, thus, enabling a distributed scalable formulation of the proposed control scheme and iv) numerically benchmark the proposed method against controllers that do not account for the grid constraints (grid-unaware local controllers (GULC) and myopic maximum power controllers (MMPC).

The rest of the paper is organised as follows. In Section II, we first introduce the nomenclature, the grid modelling and the mathematical formulation of the problem. In Section III the ADMM technique used to distribute the problem is briefly recalled with respect to its application to the proposed control framework. In Section IV, the results produced by applying the proposed method to the IEEE-34 node feeder [8] are presented. Finally, Section V concludes the paper.

\section{PRoblem Formulation}

In this work, we consider generic 3-phase distribution networks (whose phases are denoted as $a, b$ and $c$ ) with either meshed or radial topologies, where the neutral is available and distributed. They contain $N=|\mathcal{N}|$ buses, where $\mathcal{N}$ is the set of bus indexes, and, $|\mathcal{E}|$ branches with $\mathcal{E}$ the set of network branches. $\mathcal{R} \subset \mathcal{N}$ is the set of resource nodes. $\mathcal{C} \subset \mathcal{R}$ is the set of nodes to which EV CSs are connected. $\mathcal{Z} \subset \mathcal{N} \backslash \mathcal{R}$ is the set of zero-injection nodes. All resources (i.e. generation, loads and zero-injection nodes) are modelled as PQ nodes. $\{0\} \subset \mathcal{N}$ is the slack node. Assuming that different branches of the network are not electromagnetically coupled and have non-zero admittance, network branches are represented by 3-phase $\Pi$ circuit equivalents with known parameters that already embed the neutral conductor [9]. Thus ${ }^{1}$, the network is described by the so-called compound admittance matrix [10] denoted by $\overline{\boldsymbol{Y}}=\left[Y_{i j}^{\phi_{1} \phi_{2}}\right]$, with, $i, j \in \mathcal{N}$ and $\phi_{1}, \phi_{2} \in\{a, b, c\}$. The phase-to-neutral nodal voltages and nodal injected currents are respectively denoted by $\overline{\boldsymbol{E}}_{t}^{\mathrm{abc}}=\left[\bar{E}_{i, t}^{\phi}\right]$ and $\overline{\boldsymbol{I}}_{t}=\left[\bar{I}_{i, t}^{\phi}\right]$, with $t$ being the time-step, $i \in \mathcal{N}$ and $\phi \in\{a, b, c\}$. The phase-to-neutral nodal positive-, negative- and zero- sequence voltages are denoted by $\overline{\boldsymbol{E}}_{t}^{\mathrm{seq}}=\left[\bar{E}_{i, t}^{\mathrm{seq}}\right]$, with $i \in \mathcal{N}$ and seq $\epsilon$ $\{$ positive, negative, zero $\}$. Sequence voltages can be directly computed by multiplying the phase-to-ground nodal voltages with the transformation matrix $\boldsymbol{T}^{\mathrm{abc} \rightarrow \text { seq }}=\operatorname{diag}\left(\boldsymbol{T}_{i}^{\mathrm{abc} \rightarrow \text { seq }}\right)$, where $\boldsymbol{T}_{i}^{\mathrm{abc} \rightarrow \text { seq }}=\frac{1}{3}\left[1,1, \alpha ; 1, \alpha^{2}, \alpha ; 1, \alpha, \alpha^{2}\right]$, with $\alpha=$ $e^{j 2 \pi / 3}$. The branch currents are denoted by $\overline{\boldsymbol{I}}_{\mathrm{br}, t}=\left[\bar{I}_{i, j, t}^{\phi}\right]$, with $(i, j) \in \mathcal{E}$. The apparent power injections are denoted by $\overline{\boldsymbol{S}}_{t}=\boldsymbol{P}_{t}+j \boldsymbol{Q}_{t}=\left[\bar{S}_{i, t}^{\phi}\right]=\overline{\boldsymbol{s}}_{t}+\overline{\boldsymbol{s}}_{t, \mathrm{EV}}$, with $\overline{\boldsymbol{s}}_{t}=$ $\left[\mathbb{1}_{i \in \mathcal{R}}\left\{\bar{s}_{i, t}^{\phi}\right\}\right]$ being the nodal injections of all resources, $\overline{\boldsymbol{s}}_{t, \mathrm{EV}}=\left[\mathbb{1}_{i \in \mathcal{C}}\left\{\bar{s}_{i, \mathrm{EV}, t}^{\phi}\right\}\right]$ being the nodal injections of the pernode-aggregated $\mathrm{EV} \mathrm{CSs}$. The apparent power injections at the slack node are denoted by $\overline{\boldsymbol{S}}_{0, t}=\left[\bar{S}_{0, t}^{\phi}\right]=\left[\bar{E}_{0, t}^{\phi} \underline{I}_{0, t}^{\phi}\right]$ with $\phi \in\{a, b, c\}$. We adopt the convention where negative nodal injections are absorptions while positive injections are actual injections into the grid. Note that active nodal power injections of per-node-aggregated EV CSs, $\boldsymbol{p}_{t, \mathrm{EV}}=\Re\left\{\overline{\boldsymbol{s}}_{t, \mathrm{EV}}\right\}$, are nonpositive as we only consider EV charging in the scope of this paper. However, the reactive nodal power injections of pernode-aggregated EV CSs, $\boldsymbol{q}_{t, \mathrm{EV}}=\Im\left\{\overline{\boldsymbol{s}}_{t, \mathrm{EV}}\right\}$, are considered bi-directional when one or more CSs connected to a node $i \in \mathcal{C}$ support bi-directional reactive power-flows (e.g. mode 4 - DC chargers).

The goal of the proposed method is to solve an optimization problem (OP), at every time-step $t$, that determines $\overline{\boldsymbol{s}}_{t, \mathrm{EV}}$. The OP objectives and constraints can be grouped into two subproblems. The first, referred to as grid sub-problem, guarantees the safe operation of the grid by solving an OPF that considers the stochastic nature of non-EV nodal injections (c.f. II-A). The second, referred to as EV sub-problem, satisfies EV user requirements while minimizing EV-battery wearing. In the following we present the objectives and constraints of each of the sub-problems separately.

\section{A. Grid sub-problem}

The grid sub-problem is a stochastic security-constrained optimal-power-flow (OPF). As known, OPF problems are non-

\footnotetext{
${ }^{1}$ Matrices and vectors will be denoted in bold (e.g. $M$ ), complex numbers will be denoted with an over-bar (e.g. $\bar{x}=|\bar{x}| \angle \bar{x}=\Re\{x\}+j \Im\{x\}$ ), complex conjugates with a under-bar (e.g. $\underline{x}=\operatorname{conj}(\bar{x})=|\underline{x}| \angle \underline{x}=\Re\{x\}-j \Im\{x\}$ ).
} 
convex due to the nature of the power-flow equations. Thus, to make it tractable and efficiently solvable, we linearize the power flow equations to express the security constraints (see section II-A2) [11]. To deal with the stochasticity of nonEV injections, we use scenario-based optimization. Note that, even though we linearize the power-flow equations, we did not use chance-constrained optimization since our linearization model is dependent on the (stochastic) state of the grid and is not static (see [12]). Each scenario contains a collection of nodal power injections for resource nodes sampled from precomputed probability density functions (PDFs). In the following, we first describe the method to create the latter PDFs. Then, we explain the formulation of the security constraints and, finally, present the grid sub-problem objective.

1) Constructing PDFs for resources' nodal power injections: the PDFs are created using historical data in the form of active and reactive nodal injection profiles for every resource connected to a given node. Each of these profiles is first clustered into 4 seasons (i.e. winter, spring, summer and autumn), then, sub-clustered into day-type (i.e. working days, weekend or vacation days), then, finally sub-sub-clustered into the fixed time resolution of the control algorithm. For every sub-cluster a Gaussian mixed model (i.e. a sum of multivariate Gaussian normal distributions) is fitted to the data using MATLAB's function fitgmdist. This is done to account for the time correlation between the different time-steps for each specific sub-clusters. At the end, we obtain multivariate (the random variables here are all the time-steps of a given day) PDFs for each nodal injection pertaining to a season and day-type.

2) Constraints: they are the static operational and power quality constraints of a distribution grid, i.e., the branch currents magnitudes, the nodal voltages magnitudes, the ratios of nodal voltages negative- over positive- sequences and zeroover positive- sequences, and the substation apparent power magnitudes, should all be within predefined bounds. The negative- and zero- sequence voltage bounds are added to abide with the EN50160 std. [13] and to mitigate the voltage unbalances created by single-phase EV chargers. Each of the latter electrical quantities is linearized and expressed using their partial derivatives with respect to active and reactive nodal injections, i.e., sensitivity coefficients computed using the approach proposed [7]. The linearization is nothing more than a first-order Taylor expansion of the system state variables about a given set of nodal power injections. As we consider scenario-based optimization, each grid operational constraint needs to be verified for all scenarios $d=1, \ldots, D$. Recall that a scenario represents random realizations of resource injections that assume the non-presence of EV CSs. The constraints are given by,

$$
\begin{gathered}
\left|\overline{\boldsymbol{E}}_{t}^{\mathrm{abc}}\right|^{d} \in\left[E^{\mathrm{nom}}-\beta, E^{\text {nom }}+\beta\right],\left|\overline{\boldsymbol{I}}_{\mathrm{br}, t}\right|^{d} \leq \boldsymbol{I}_{\mathrm{br}}^{\mathrm{max}},\left|\overline{\boldsymbol{S}}_{0, t}\right|^{d} \leq \boldsymbol{S}_{0}^{\mathrm{max}} \\
\left|\overline{\boldsymbol{E}}_{t}^{\text {negative }}\right|^{d} \leq \delta_{\text {neg }}\left|\overline{\boldsymbol{E}}_{t}^{\text {positive }}\right|^{d},\left|\overline{\boldsymbol{E}}_{t}^{\text {zero }}\right|^{d} \leq \delta_{\text {zer }}\left|\overline{\boldsymbol{E}}_{t}^{\text {positive }}\right|^{d}
\end{gathered}
$$

where, $E^{\text {nom }}$ and $\beta$ are tolerances for the nodal voltage magnitudes, $\delta_{\text {neg }}$ and $\delta_{\text {zer }}$ are, respectively, tolerances for the magnitudes of the negative- and zero- sequence nodal voltages, $\overline{\boldsymbol{I}}_{\mathrm{br}}^{\max }=\left[\bar{I}_{i, j, t}^{\phi, \max }\right]$, with $(i, j) \in \mathcal{E}$, is the vector of branch am- pacity limits, $\overline{\boldsymbol{S}}_{0}^{\max }=\left[\bar{S}_{0, t}^{\phi, \max }\right]$ is the vector of per phase substation transformer apparent power limits and a superscript $d$ refers to a scenario. $\left|\overline{\boldsymbol{E}}_{t}^{\text {abc }}\right|,\left|\overline{\boldsymbol{I}}_{\text {br }, t}\right|,\left|\overline{\boldsymbol{S}}_{0, t}\right|,\left|\overline{\boldsymbol{E}}_{t}^{\text {negative }}\right|,\left|\overline{\boldsymbol{E}}_{t}^{\text {zero }}\right|$ and $\left|\overline{\boldsymbol{E}}_{t}^{\text {positive }}\right|$ are generically linearly approximated by,

$$
\begin{aligned}
\boldsymbol{\Gamma}_{t}^{d} \approx \tilde{\boldsymbol{\Gamma}}_{t}^{d}\left(\tilde{\overline{\boldsymbol{S}}}_{t}^{d}\right) & +\left.\frac{\partial \boldsymbol{\Gamma}_{t}}{\partial \boldsymbol{P}_{t}}\right|_{\tilde{\overline{\boldsymbol{S}}}_{t}^{d}}\left(\boldsymbol{p}_{t, \mathrm{EV}}-\boldsymbol{p}_{t-1, \mathrm{EV}}\right) \\
& +\left.\frac{\partial \boldsymbol{\Gamma}_{t}}{\partial \boldsymbol{Q}_{t}}\right|_{\tilde{\overline{\boldsymbol{S}}}_{t}^{d}}\left(\boldsymbol{q}_{t, \mathrm{EV}}-\boldsymbol{q}_{t-1, \mathrm{EV}}\right)
\end{aligned}
$$

where, $\boldsymbol{\Gamma} \in\left\{\left|\overline{\boldsymbol{E}}_{t}^{\text {abc }}\right|,\left|\overline{\boldsymbol{I}}_{\text {br }, t}\right|,\left|\overline{\boldsymbol{S}}_{0, t}\right|,\left|\overline{\boldsymbol{E}}_{t}^{\text {negative }}\right|,\left|\overline{\boldsymbol{E}}_{t}^{\text {zero }}\right|\right.$, $\left.\left|\overline{\boldsymbol{E}}_{t}^{\text {positive }}\right|\right\}, \tilde{\boldsymbol{\Gamma}}_{t}^{d}\left(\tilde{\overline{\boldsymbol{s}}}_{t}^{d}\right)$ is the electrical quantity resulting from a load-flow computation with nodal injections $\tilde{\overline{\boldsymbol{S}}}_{t}^{d}=\tilde{\overline{\boldsymbol{s}}}_{t}^{d}+$ $\overline{\boldsymbol{s}}_{t-1, \mathrm{EV}}$, where $\tilde{\overline{\boldsymbol{s}}}_{t}^{d}$ are the sampled scenarios and $\overline{\boldsymbol{s}}_{t-1, \mathrm{EV}}$ the optimal EV injections of the previous control timestep, and, the sensitivity coefficients $\partial \boldsymbol{\Gamma}_{t} /\left.\partial \boldsymbol{P}_{t}\right|_{\tilde{\boldsymbol{S}}_{t}^{d}}$ and $\partial \boldsymbol{\Gamma}_{t} /\left.\partial \boldsymbol{Q}_{t}\right|_{\tilde{\boldsymbol{S}}_{t}^{d}}$ being, respectively, the partial derivatives of the electrical quantity $\Gamma$ with respect to nodal active and reactive power injections, computed using the injections $\tilde{\overline{\boldsymbol{S}}}_{t}^{d}$ following the method in [7]. Note that in [7] only the techniques for computing the nodal voltage and branch currents sensitivity coefficients are presented. However, assuming that $\overline{\boldsymbol{Y}}$ is invariant to nodal injections, all other sensitivity coefficients can be directly computed using the following (3) and equation (10) in [7].

$$
\begin{gathered}
\left.\frac{\partial \bar{S}_{0, t}^{\phi}}{\partial\{\boldsymbol{P} \text { or } \boldsymbol{Q}\}_{t}}\right|_{\tilde{\boldsymbol{s}}_{t}^{d}}=\left.\frac{\partial \bar{E}_{0, t}^{\phi}}{\partial\{\boldsymbol{P} \text { or } \boldsymbol{Q}\}_{t}}\right|_{\tilde{\tilde{\boldsymbol{s}}}_{t}^{d}} \bar{I}_{0, t}^{\phi}+\left.\bar{E}_{0, t}^{\phi} \frac{\partial \bar{I}_{0, t}^{\phi}}{\partial\{\boldsymbol{P} \text { or } \boldsymbol{Q}\}_{t}}\right|_{\tilde{\overline{\boldsymbol{s}}}_{t}^{d}} \\
\left.\frac{\partial\left[\bar{E}_{i, t}^{\text {zero }}, \bar{E}_{i, t}^{\text {positive }}, \bar{E}_{i, t}^{\text {negative }}\right]^{T}}{\partial\{\boldsymbol{P} \text { or } \boldsymbol{Q}\}_{t}}\right|_{\tilde{\boldsymbol{s}}_{t}^{d}}=\left.\boldsymbol{T}_{i}^{\text {abc } \rightarrow \operatorname{seq}} \frac{\partial\left[\bar{E}_{i, t}^{a}, \bar{E}_{i, t}^{b}, \bar{E}_{i, t}^{c}\right]^{T}}{\partial\{\boldsymbol{P} \text { or } \boldsymbol{Q}\}_{t}}\right|_{\tilde{\overline{\boldsymbol{s}}}_{t}^{d}}
\end{gathered}
$$

Finally, note that the only variables in (2) for the control timestep $t$, i.e., $\boldsymbol{p}_{t, \mathrm{EV}}$ and $\boldsymbol{q}_{t, \mathrm{EV}}$, do not have a superscript $d$ as we want these variables to satisfy the constraints for all scenarios, rendering the satisfaction of the constraints robust to nodal injections stochasticity as $D$ increases.

3) Objective: From the grid operator perspective, since all the security constraints were added as hard constraints, the objective is, in theory, free to be defined by the modeller. In practice, having branch currents close to their ampacity limits can affect the cables' lifetimes and increase network losses, thus, the simplest considered objective is to minimize

$$
\left\langle\mathbf{1},\left(\left.\frac{\partial\left|\overline{\boldsymbol{I}}_{\mathrm{br}, t}\right|}{\partial \boldsymbol{Q}_{t}}\right|_{\tilde{\tilde{S}}_{t}^{d}}\left(\boldsymbol{q}_{t, \mathrm{EV}}-\boldsymbol{q}_{t-1, \mathrm{EV}}\right)\right) \oslash\left|\overline{\boldsymbol{I}}_{\mathrm{br}, t}\right|\right\rangle,
$$

where 1 is a vector of ones, $\oslash$ is the Hadmarad division (i.e. element-wise vector division) and the $\langle$,$\rangle is the dot product.$ The latter objective minimises the per-unit-amapcity branchcurrent-magnitudes through the reactive power contribution at EV CSs. This is done to allow the active power to be fully optimised for the EV sub-problem objectives (see section II-B2), thus, not penalising EV charging.

\section{B. EV sub-problem}

The EV CSs sub-problem aims, for every time-step, to compute the power allocations given to the different EVs connected 
to the different CSs $k$, with $k=1, \ldots, K(i)$, where $K(i)$ is the total number of CSs connected to node $i \in \mathcal{C}$. Indeed, we assume that at every node $i \in \mathcal{C}$, there is an aggregator that can send active and reactive power setpoints to all CSs (or EV plugs) $k=1, \ldots, K(i)$. For simplicity, and without loss of generality, each CS is assumed to have a fixed charging mode, i.e. three-phase (3ph), single-phase $(1 \mathrm{ph})$ or direct current charging (DC). Furthermore, we assume three-phase chargers are balanced, DC chargers are connected to all three phases through a fully balanced inverter (i.e. balanced in active and reactive powers), and single-phase chargers can be connected to any phase. Additionally, only CSs with DC charging are assumed to have controllable bidirectional reactive power flow as this is the situation in practice. However, in the future, if other charger types start supporting bidirectional power flow, little change needs to be done to the problem formulation as it remains fundamentally the same ${ }^{2}$.

To formulate the problem, we assume that every time an $\mathrm{EV}$ is plugged to a CS, the user will input five quantities: (i) the initial $\mathrm{SoC}$ of the vehicle in percentage $\left(\mathrm{SoC}_{t_{0, k, i}, k, i}\right.$, with $t_{0, k, i}$ being the arrival time of the car at the $k$-th CS of node $i$ ), (ii) the estimated departure time $\left(t_{f, k, i}\right)$, (iii) the desired $\mathrm{SoC}$ at departure $\left(\mathrm{SoC}_{t_{f, k, i}, k, i}\right)$, (iv) the car model, and, (v) whether or not the user would like the $k$-th CS to continue providing charging power if the time $t$ exceeds $t_{f, k, i}$ $\left(\tau_{t, k, i}^{+} \in\{0,1\}\right)$. Note that, in practice, the departure time can be chosen by the user if he/she actually has a time-constraint, otherwise, the charging duration can be translated to the user as an equivalent charging price. It is worth mentioning that the car model provides information on the maximum charging powers $\left(P_{k, i}^{\max }>0\right)$ and battery capacity $\left(E_{k, i}^{\max }\right)$ of the vehicle, the charging efficiency $\left(\eta_{k, i}\right)$ and whether or not the SoC is measured (and sent) by the vehicle (to the CS). In the following we will present the constraints and objectives pertaining to the EV CSs sub-problem.

1) Constraints: the constraints of each EV aggregator of node $i \in \mathcal{C}$ ensure three aspects: (i) the $\mathrm{SoC}$ of each $\mathrm{EV}$ connected to the $k$-th CS of node $i$ will remain below the target SoC; (ii) the per-phase nodal injections correspond to the charging mode of each connected EV, and, (iii) charging powers are all within EV inverter limitations. The constraints are described here below.

SoC lower that target after application of optimal EV power setpoint

$$
\mathrm{SoC}_{t, k, i}-\frac{\eta_{k, i} P_{t, k, i} \Delta t}{E^{\max }} \leq \operatorname{SoC}_{t_{f, k, i}, k, i}
$$

EV power setpoint limitations

$$
\left(P_{t, k, i}, Q_{t, k, i}\right) \in \begin{cases}\left\{(P, Q) \mid P^{2}+Q^{2}<S_{k, i}^{\max 2},\right. & \omega_{t, k, i}=1 \\ \left.P \geq-P_{k, i}^{\max }\right\} & \\ \{(0,0)\} & \omega_{t, k, i}=0\end{cases}
$$

\footnotetext{
${ }^{2}$ Extending the method to support Vehicle-to-Grid-enabled charged is straight-forward using the techniques presented in [14].
}

Translation of EV power setpoint to phase powers

$$
\begin{aligned}
& P_{t, k, i}= \begin{cases}P_{t, k, i}^{a}+P_{t, k, i}^{b}+P_{t, k, i}^{c} & k \in 3 \text { ph or DC } \\
P_{t, k, i}^{\phi} & k \in 1 \mathrm{ph}, \phi \in\{\end{cases} \\
& Q_{t, k, i}=\left\{\begin{array}{l}
Q_{t, k, i}^{a}+Q_{t, k, i}^{b}+Q_{t, k, i}^{c} \\
0
\end{array} \quad k \in \mathrm{DC}\right. \\
& P_{t, k, i}^{a}=P_{t, k, i}^{b}=P_{t, k, i}^{c}, P_{t, k, i}^{a} \leq 0 \\
& Q_{t, k, i}^{a}=Q_{t, k, i}^{b}=Q_{t, k, i}^{c} \\
& P_{t, k, i}^{b}=P_{t, k, i}^{c}=0, P_{t, k, i}^{a} \leq 0 \\
& k \in 1 \text { ph or } 3 \text { ph } \\
& k \in 3 \text { ph or DC } \\
& k \in \mathrm{DC} \\
& k \in 1 \mathrm{ph}
\end{aligned}
$$

Link to Grid sub-problem Variables

$$
\bar{s}_{i, \mathrm{EV}, t}^{\phi}=\sum_{k=1}^{K(i)} P_{t, k, i}^{\phi}+j Q_{t, k, i}^{\phi}, \forall \phi \in\{a, b, c\}
$$

where, $\Delta t$ is the fixed control time-step resolution, a subscript $t$ represents time-step $t, P_{t, k, i}$ and $Q_{t, k, i}$ are variables representing respectively the active and reactive total power at the $k$-th CS, $P_{t, k, i}^{a}, P_{t, k, i}^{b}, P_{t, k, i}^{c}, Q_{t, k, i}^{a}, Q_{t, k, i}^{b}$ and $Q_{t, k, i}^{c}$ are variables representing the active and reactive per phase nodal injections at the $k$-th $\mathrm{CS}, \omega_{t, k, i} \in\{0,1\}$ is equal to 1 if an EV is plugged at time-step $t$ to the $k$-th $\mathrm{CS}$ of node $i \in \mathcal{C}$ and $S_{k, i}^{\max }$ is the maximum supported apparent power of the $k$-th CS of node $i$ that is here assumed to be independent of the AC and DC-side voltages of each CS. Also, for simplicity, the maximum charging power $P_{k, i}^{\max }$ per $\mathrm{EV}$ and associated efficiency are assumed independent of the SoC which, in practice, may not be true as some EVs reduce their charging powers when their SoC gets close to 1 . However, if known, these dependencies can easily be added to the problem formation. Note that, the boolean $\omega_{t, k, i} \in\{0,1\}$ is known at every time-step which simplifies the problem as it does not introduce the non-convexity usually associated with integer variables representing EV arrival and departure times. Finally, if the $\mathrm{SoC}$ of the present time-step $t\left(S o C_{t, k, i}\right)$ is measured then we directly have access to it, otherwise, it is estimated as:

$$
\operatorname{SoC}_{t, k, i}=\operatorname{SoC}_{t-1, k, i}-\frac{\omega_{t, k, i} \eta_{k, i} P_{t-1, k, i} \Delta t}{E_{k, i}^{\max }}
$$

2) Objective: The objective of the EV sub-problem is twofold.

The first objective is to fairly achieve the target SoC for every connected EV as soon as possible. This is given by,

$$
\sum_{k=1}^{K(i)} \kappa_{t, k, i}\left(\operatorname{SoC}_{t_{f, k, i}, k, i}-\operatorname{SoC}_{t, k, i}+\frac{\eta_{k, i} P_{t, k, i} \Delta t}{E^{\text {max }}}\right)
$$

where $\kappa_{t, k, i}$ is given by

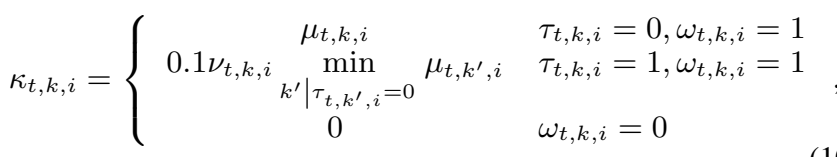

where $\tau_{t, k, i}$ is a boolean constant that is true if the present time-step $t$ exceeds the $\mathrm{k}^{\text {th }}$ EV's departure time $\left(t_{f, k, i}\right)$, $\mu_{t, k, i} \in[0,1]$ is given by,

$$
\mu_{t, k, i}=\frac{\min _{k^{\prime} \mid \tau_{t, k^{\prime}, i}=0}\left\{t_{f, k^{\prime}, i}-t\right\}}{t_{f, k, i}-t} \frac{\operatorname{SoC}_{t_{f}, k, i}-\operatorname{SoC}_{t, k, i}}{\max _{k^{\prime} \mid \tau_{t, k^{\prime}, i}=0}\left\{\operatorname{SoC}_{t_{f}, k^{\prime}, i}-\operatorname{SoC}_{t, k^{\prime}, i}\right\}}
$$


and $\nu_{t, k, i} \in[0,1]$ is given by,

$$
\mu_{t, k, i}=\frac{\operatorname{SoC}_{t_{f}, k, i}-\operatorname{SoC}_{t, k, i}}{\max _{k^{\prime} \mid \tau_{t, k^{\prime}, i}=1}\left\{\operatorname{SoC}_{t_{f}, k^{\prime}, i}-\operatorname{SoC}_{t, k^{\prime}, i}\right\}} .
$$

The weighting factor $\kappa_{t, k, i}$ gives: (i) more weight to plugged EVs with SoCs that are far from their target SoCs $\left(S O C_{t_{f}, k, i}\right)$ and with little remaining time before their specified departure times $\left(t_{f, k, i}\right) \quad\left[\mu_{t, k, i}\right]$ (ii) ten times less weight than the least weighted plugged EV that has not surpassed its $t_{f, k, i}$ to plugged EVs that have surpassed their $t_{f, k, i}$ $\left[0.1 \min _{k \mid \tau_{t, k}, i=0} \mu_{t, k, i}\right]$ (iii) more weight, within plugged EVs that have surpassed their $t_{f, k, i}$, to plugged EVs with SoCs that are far from their target SoC $\left(S O C_{t_{f}, k, i}\right)\left[\nu_{t, k, i}\right]$.

The second objective is to minimize EV battery wearing. As suggested in [15], the latter can be achieved by avoiding frequent variations in EV charging power. This is given by,

$$
\sum_{k=1}^{K(i)} \lambda_{t, k, i} \kappa_{t, k, i}\left|P_{t, k, i}-P_{t-1, k, i}\right| \frac{\Delta t}{E^{\max }},
$$

where the scaling factor $\lambda_{t, k, i} \in[0,0.5]$, given by,

$$
\lambda_{t, k, i}=\frac{1}{2} \frac{\sum_{t^{\prime}=t_{0, k, i}+1}^{t-1}\left|P_{t^{\prime}, k, i}-P_{t^{\prime}-1, k, i}\right|}{\sum_{t^{\prime}=t_{0, k, i}+1}^{t-1} P_{k, i}^{\max }}
$$

gives more weight to (i.e. penalizes more) EVs whose charging powers have changed several times since being plugged. In the worst case where the charging power varies between each consecutive time-steps between 0 and $P_{k, i}^{\max }$ since the EV was plugged, $\lambda_{t, k, i}$ will be equal to 0.5 .

As a final note, observe that both (9) and (13) are of the same nature, i.e. they are all in percentages, and, are scaled by $\kappa_{t, k, i}$. This is done to ensure that for any EV, its battery ageing objective can be weighted, thanks to $\lambda_{t, k, i}$, from zero to half its $\mathrm{ScC}$ objective.

\section{Centralised Full Optimization Problem}

The centralised full optimization problem is given by,

$$
\begin{array}{ll}
\min _{\boldsymbol{p}_{t, \mathrm{EV}}, \boldsymbol{q}_{t, \mathrm{EV}}} \sigma_{\text {grid }}((4))+\sigma_{\mathrm{EV}} \sum_{i \in \mathcal{C}}((9)+(13)) \\
\text { s.t. } \\
\begin{array}{ll}
(1), & \forall d=1, \ldots, D \\
\text { (5) }-(7), & \forall k=1, \ldots, K(i), \forall i \in \mathcal{C} \\
(8), & \forall \phi \in\{a, b, c\}, \forall i \in \mathcal{C}
\end{array}
\end{array}
$$

where $\sigma_{\text {grid }}$ and $\sigma_{\mathrm{EV}}$ are weights. Note that, minimizing (13) smooths the EV active power charging profiles but also renders the constraints (1) less prone to approximation errors. This is because the less nodal injection changes there are between successive iterations, the more the linearizations in (1) are accurate.

\section{DistRiBUted OPTIMIZATION}

Solving the centralized problem (15) requires user information from local EV aggregators, which might not be easily accessible due to privacy and security concerns among the EV owners. Also, the centralized approach might not be easily scalable with respect to number of EV CSs. Thus, following the approach in [16], we reformulate the centralized optimization problem (15) by introducing a set of auxiliary variables $\bar{s}_{i, \mathrm{EV}, t}^{\prime \phi}, \forall \phi \in\{a, b, c\}$, which mimic the behaviour of original variables $\bar{s}_{i, \mathrm{EV}, t}^{\phi}, \forall \phi \in\{a, b, c\}$. The augmented problem is given by,

$$
\begin{aligned}
& \min _{\boldsymbol{p}_{t, \mathrm{EV}}, \boldsymbol{q}_{t, \mathrm{EV}}, \boldsymbol{p}_{t, \mathrm{EV}}^{\prime}, \boldsymbol{q}_{t, \mathrm{EV}}^{\prime}} \sigma_{\text {grid }}((4))+\sigma_{\mathrm{EV}} \sum_{i \in \mathcal{C}}((9)+(13)) \\
& \text { s.t. } \\
& \text { (1), } \\
& \text { (5) }-(7) \text {, } \\
& \bar{s}_{i, \mathrm{EV}, t}^{\prime \phi}=\sum_{k=1}^{K(i)} P_{t, k, i}^{\phi}+j Q_{t, k, i}^{\phi}, \quad \forall \phi \in\{a, b, c\}, \forall i \in \mathcal{C} \\
& \overline{\boldsymbol{s}}_{t, \mathrm{EV}}=\overline{\boldsymbol{s}}_{t, \mathrm{EV}}^{\prime}
\end{aligned}
$$

The problem in (16) is a standard sharing problem that is separable in each component $\bar{s}_{i \mathrm{EV}, t}^{\prime \phi}, \forall \phi \in\{a, b, c\}$. Using a sequence of Lagragian multipliers $\boldsymbol{y}_{t}$ and the scaled Lagragian multipliers $\boldsymbol{u}_{t}=\boldsymbol{y}_{t} / \rho$, the last constraint of (16) can be moved into the objective. The reformulated problem can be solved using Algorithm 1 that exploits the iterative scaled-ADMM sharing problem [17].

Algorithm 1 is iterative, with each iteration $v$ composed of three steps. First, each EV aggregator associated to node $i \in \mathcal{C}$ solves, separately and in parallel, (17), then sends the solutions to the grid aggregator. Once the grid aggregator receives the solutions of all EV aggregators, it solves (18) and updates the dual variables with (19). Finally, the grid aggregator sends back to the distributed EV aggregators the corresponding dual variables and its optimal solution (i.e. $\bar{s}_{i, \mathrm{EV}, t} \phi^{v}, \forall \phi \in\{a, b, c\}$ where the supercript $v$ entails a value at the $v$-th iteration). The procedure is repeated until the convergence criterias are met, i.e. the primal ( (20) ) and dual ( (21) ) residual norms are below, respective, tolerance limits $\epsilon^{\text {pri }}$ and $\epsilon^{\text {dual }}$. Additionaly, the tuning parameter $\rho^{v}$ is adaptively updated using the technique presented in chapters 3.3-3.4 in [17]. Finally, as (16) is linear, Algorithm 1 is guaranteed, in principle, to converge to the optimal solution of (16) [17].

\section{Comparative Simulation}

In order to illustrate the proposed method, a performance comparison between three distributed control algorithms is done. The first controller is the grid-aware distributed controller (GADC) that uses all the steps in Algorithm 1 with $\sigma_{\text {grid }}=0.1$ and $\sigma_{\mathrm{EV}}=10$. The second is a grid-unaware local controller (GULC) that only solves the EV aggregator local control problems (EV sub-problem) developed in this paper, i.e. for each $\mathrm{EV}$ aggregator, $\forall i \in \mathcal{C}$, (17) is solved locally without the augmented Lagrangian term in the objective (i.e. with objectives $(9)+(13)$ subject to the same constraints). The third is a myopic maximum power controller (MMPC) that simply chargers any plugged EVs at maximum power until they reach 


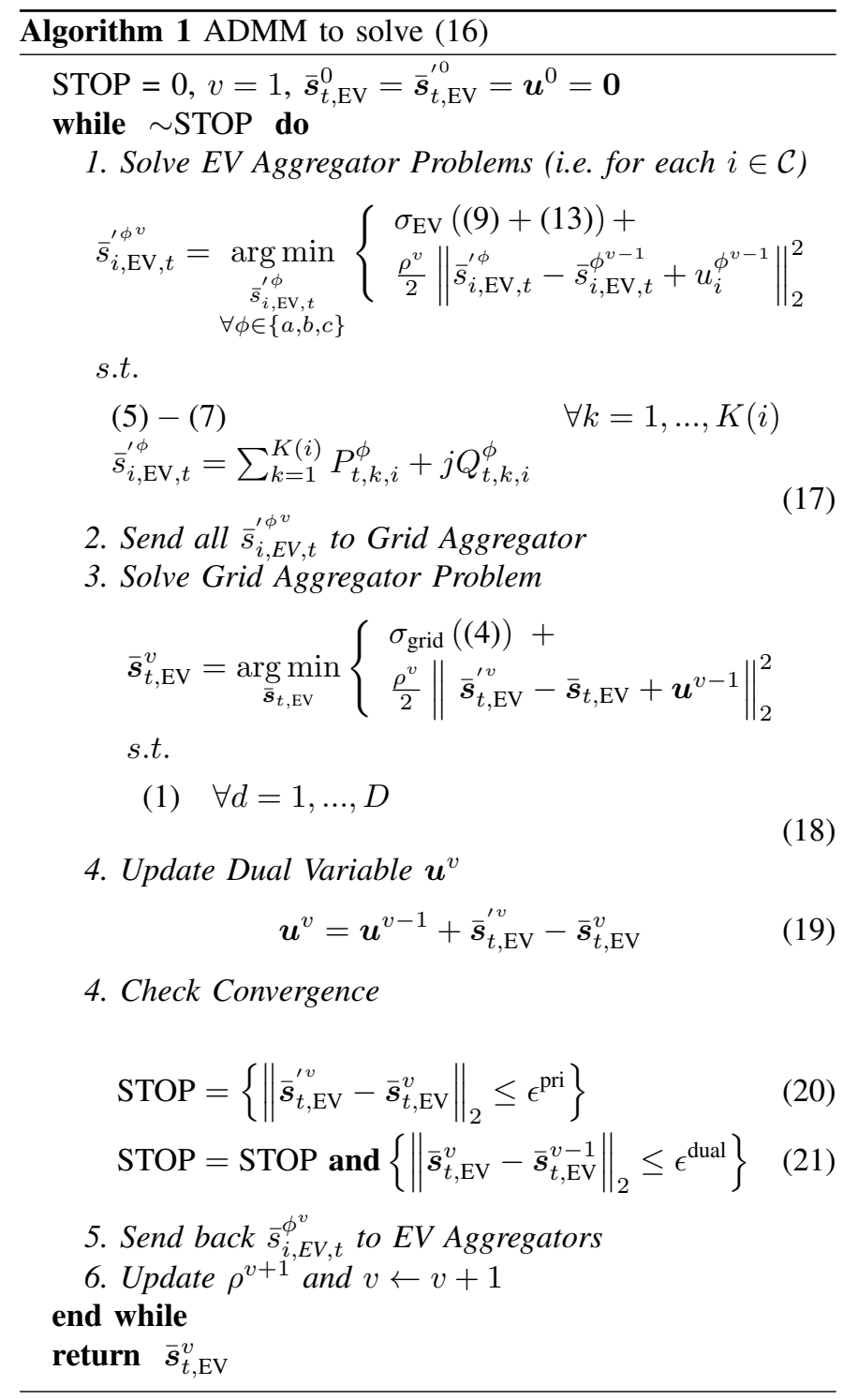

their target SoCs. Note that both grid-unaware controllers only find nodal active power EV injections and set reactive power EV injections to zero as they do not have any incentive to inject/absorb reactive power. The performance comparison consists in finding first the optimal $\mathrm{EV}$ injections computed by each controller, then, performing Monte-Carlo simulations for each controller, using the latter optimal injections, to compute the probabilities to violate grid operational and power quality constraints. Specifically, the simulation consists in performing a 24 hour (from 8:00AM to 7:55AM the next day) control run with a 5 minute control resolution (i.e. $\Delta t=5$ mins) for a summer weekday. At every time-step, the three controllers first solve their EV power allocation problems (i.e. find $\bar{s}_{t, \mathrm{EV}}$ ) using the same inputs (i.e. initial EV SoC, arrival/departure times and car model for all three controllers, and injection scenarios for the GADC controller). Then, for the same timestep, for each controller, a Monte-Carlo load-flow simulation (MCLFS) with EV injections fixed to the controller optimum is performed. All three MCLFS pertaining to the same time-step use the same newly (i.e. different than the input scenarios used to solve the GADC problem of the same time-step) randomly sampled scenarios for non-EV nodal power injections. Recall that, MCLFS consists in carrying out a large number of loadflow computations with different nodal power injections and outputs cumulative distributions functions (CDFs) of the grid state variables. To recap: the output of the simulation for each controller is, therefore, the optimal EV power allocations for all EV aggregators along with their performance vis-a-vis (i) grid operational and power quality constraints violations under stochastic non-EV injections, and (ii) meeting EV user needs (i.e. reaching target $\mathrm{SoC}$ at $\mathrm{EV}$ unplugging).

\section{A. Grid model and data used in the simulation}

The simulation is done the on the IEEE34 feeder [8], where the voltage regulators are omitted in order to worsen voltage control problems. Fig. 1 shows the network topology and resource allocation. It is worth mentioning that in this simulation $50 \%, 25 \%$ and $25 \%$ of single-phase EV chargers are respectively connected to phases $\mathrm{a}, \mathrm{b}$ and $\mathrm{c}$ of the electrical grid. The base voltage and power are respectively set to $24.9 \mathrm{kV}$ and $1 \mathrm{MVA}$. The grid security bounds are set to $E^{\text {nom }}=1$ p.u., $\beta=0.05, \delta_{\text {inv }}=0.5 \%, \delta_{\text {hom }}=0.75 \%$, $S_{0}^{\max }=1.5$ p.u. and the amapcity limits found in [8] scaled by a factor of 6. Fig. 2 shows all the aggregated (i.e. summed over all nodes and phases) non-EV load (negative) and generation (in the form of postive PV injections) daily active power profiles already sub-clustered for a summer weekday. Each aggregated profile is first disaggregated to different nodes by simply scaling the profiles in Fig. 2 using the maximum active nodal power injection limits shown in Table I. With all the disaggregated profiles, for every node, for every time-step, a multivariate PDF is computed using the technique explained in II-A1. These PDFs are used to generate the non-EV active power injection scenarios for the GADC controller and the MCLFS. Indeed, using the scenario-reduction technique presented in [18], the number of scenarios $D$ used by the GADC controller at every time-step is set to 75 and the number of load-flow computations used in each MCLFS is set to 250. Note that, reactive power injection scenarios are inferred from the active power injection scenarios using power factors sampled from the typical ranges $\left(\left[P F^{\min }, P F^{\max }\right]\right)$ given in Table I. Furthermore, to get the injections per phase, the total three phase nodal sampled active and inferred reactive injection scenarios are split pseudo-equally with an intra-phase unbalance randomly chosen between 0 and $20 \%$. Finally, note that the values in Table I were chosen in a way to create no grid operational and power quality constraint violations when EV injections are null.

\section{B. EV users model and data used in the simulation}

Even though the method is built to accommodate any random EV user behaviours (i.e. random arrival/departure time, target SoC and car model), in this simulation, they are synthesized to stress test the algorithm. Building on that, 


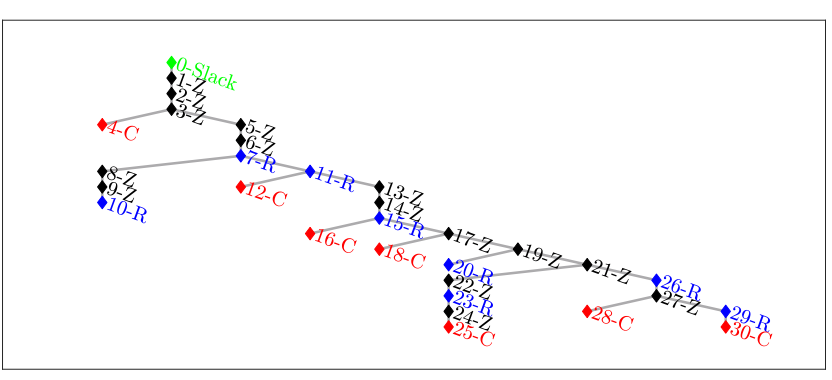

Fig. 1. Network graph with resource placement. $\mathrm{R}$ is a resource node. $\mathrm{Z}$ is a zero-injection node. $\mathrm{C}$ is a resource node also containing EV CSs.

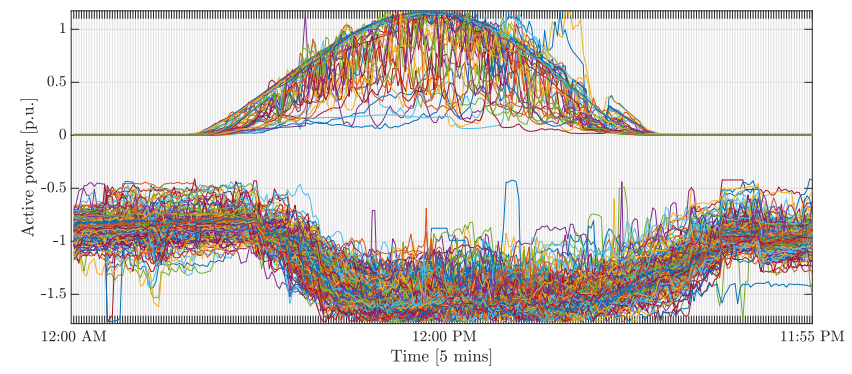

Fig. 2. Aggregated PV (positive) and load (active) active power profiles for the simulated summer weekday.

we categorize all CSs connected to a node $i \in \mathcal{C}$ into three groups: home, work and roadside. Home chargers are considered as CSs placed in residential buildings/areas that only support AC charging (i.e. three-phase (AC 3ph) and/or single-phase (1ph)). Work chargers are considered as CSs placed in industrial buildings/areas that, also, only support AC charging. Roadside chargers, however, are considered to only support DC charging. We assume that, all vehicles connected to single-phase AC chargers are Jaguar i-Pace, all vehicles connected to three-phase AC chargers are Tesla Model S, and, all vehicles connected to DC chargers are Nissan Leaf Plus. This is done to include real EV charging efficiencies, maximum powers and battery energy capacities in the simulation (see Tab. IIIa for values). Depending on

TABLE I

MAXIMUM THREE PHASE NODAL INJECTIONS AND POWER FACTOR RANGE PER NON-EV RESOURCE.

\begin{tabular}{c|c|c|c} 
Node & Max [ kW ] & $P F^{\min }$ & $P F^{\max }$ \\
\hline 7 & 112.500 & \pm 0.800 & \pm 0.867 \\
15 & 262.500 & \pm 0.800 & \pm 0.867 \\
18 & 337.500 & \pm 0.800 & \pm 0.867 \\
23 & 262.500 & \pm 0.800 & \pm 0.867 \\
25 & 187.500 & \pm 0.800 & \pm 0.867
\end{tabular}

(a) PV Generation Nodes

\begin{tabular}{c|c|c|c} 
Node & Max [ kW ] & $P F^{\min }$ & $P F^{\max }$ \\
\hline 10 & 157.500 & 0.930 & 1.000 \\
11 & 315.000 & 0.930 & 1.000 \\
20 & 472.500 & 0.930 & 1.000 \\
26 & 525.000 & 0.930 & 1.000 \\
29 & 288.750 & 0.930 & 1.000
\end{tabular}

(b) Load Nodes the group, the synthesized EV user behaviours are different. Namely, we assume that home chargers are only active at night from 5:00PM to 7:55AM the next day, a quarter of the work chargers are active from 8:30AM to 7:00PM as they are considered reserved for company employees while the rest are active from 9:00AM to 5:30PM as they are considered reserved for visitors, and roadside chargers have two charging sessions, respectively, from 8:00AM to 10:00AM (i.e. fast charging before arriving at work) and 12:00PM to 7:00PM (i.e. afternoon fast charging). The number of CSs per node, the charging modes, the exact arrival and departure EV times and, the initial and target EV battery SoCs are all randomly sampled from the ranges given in Tables IIIb-IIIc. In Tables IIIb-IIIc we distinguish between planned departure time $\left(t_{f, k, i}\right)$ and actual departure time to account for users that keep their EVs plugged after their announced departure times; we assume that this behaviour is only present for work and roadside chargers as users charging at home usually leave punctually to go to work every morning during a weekday.

\section{Results of the simulation}

1) From the grid perspective: Figs. 3 and 4 show the post-MCLFS probabilities to violate, respectively, the grid operational and power quality constraints for all time steps and control algorithms used in the simulation. It can be observed that the GADC algorithm guarantees close-to-no violations. Indeed, minor violations arise as power-flow equations are linearised which entails discrepancies between the linearised state and the real one. On the other hand, the GULC and MMPC optimal EV nodal power injections create several violations. When it comes to grid operational constraints, Fig. 3a, Fig. $3 b$ and Fig. 3c show that, respectively, nodal voltage magnitude, branch current magnitude and slack node apparent power violations are practically guaranteed for both gridunaware controllers during night-time home charging. When it comes to power quality, for both grid-unaware controllers, the negative- and zero-sequence components constraints are also violated mostly at night. This is explained by the presence of a large number of users that plug their single-phase EVs into the grid and thus creating high voltage unbalances. Even though it still performs badly, it can be observed that the GULC controller sometimes incurs lower constraint violation probabilities compared to the MMPC controller. This can be explained by the smoothing effect of EV charging that arises due to the GULC objectives. In summary, from Figs. 3 and 4, it is safe to conclude that grid-unaware EV charging largely jeopardizes the safe operation of the grid.

2) From the EV users perspective: Fig. 5 shows, using a boxplot representation, the difference between all target SoCs and the actual ones at time of departure. Fig. 5a represents a base-case as the MMPC algorithm charges EVs at full power. The reason why there are some unachieved charging targets is due to the fact that initial and desired SoCs are randomly sampled. Thus sometimes it is simply not possible to achieve the desired SoC during the time window where the EV is plugged. Fig. 5b shows that the GULC controller behaves similarly 
TABLE II

EV SIMULATED ENVIRONEMENT

\begin{tabular}{c|c|c|c|c} 
Charging Mode & Car Model & $P_{k, i}^{\max }[\mathrm{kW}]$ & $E_{k, i}^{\max }[\mathrm{kWh}]$ & $\eta_{k, i}$ \\
\hline AC 1ph & Jaguar iPace & 7.4 & 84.7 & 0.92 \\
\hline AC 3ph & Tesla Model S & 17 & 100 & 0.85 \\
\hline DC & Nissan Leaf Plus & 50 & 64 & 0.95 \\
\hline
\end{tabular}

(a) EV considered car models

\begin{tabular}{c|c|c|c|c} 
Nodes & Category & Charging Mode & $S_{k, i}^{\max }[\mathrm{kVA}]$ & $K(i)$ \\
\hline$\{30,28,25\}$ & Home & AC 1 ph or AC $3 \mathrm{ph}$ & 3.3 or 7.4 & {$[60 ; 120]$} \\
\hline$\{16,12\}$ & RoadSide & DC & 50 & {$[40 ; 60]$} \\
\hline$\{4,18\}$ & Work & AC 1 ph or AC 3ph & 7.4 or 17 & {$[50 ; 100]$} \\
\hline
\end{tabular}

(b) EV user synthesized behaviour

\begin{tabular}{|c|c|c|c|c|c|}
\hline Nodes & $t_{0, k, i}$ & $t_{f, k, i}$ & Actual Departure Time & $S O C_{t_{0, k, i}, k, i}$ & $S O C_{t_{f, k, i}, k, i}$ \\
\hline$\{30,28,25\}$ & [5:00PM; 11:00PM] & [6:00AM; 7:55AM] & $t_{f, k, i}$ & {$[0.15 ; 0.45]$} & {$[0.95 ; 1.00]$} \\
\hline$\{16,12\}$ & $\begin{array}{ll}\text { Session } 1- & {[8: 05 \mathrm{AM} ; 10: 00 \mathrm{AM}]} \\
\text { Session } 2- & {[12: 00 \mathrm{PM} ; 7: 00 \mathrm{PM}]}\end{array}$ & $\begin{array}{l}\text { [8:35AM; } 11: 00 \mathrm{AM}] \\
{[12: 35 \mathrm{PM} ; 8: 00 \mathrm{PM}]}\end{array}$ & $t_{f, k, i}+[0-20] \operatorname{mins}$ & $\begin{array}{l}{[0.15 ; 0.35]} \\
{[0.10 ; 0.35]}\end{array}$ & {$[0.60 ; 0.80]$} \\
\hline$\{4,18\}$ & $\begin{array}{cc}\text { Visitors- } & {[9: 00 \mathrm{AM} ; 5: 30 \mathrm{PM}]} \\
\text { Employees- } & {[8: 30 \mathrm{AM} ; 11: 00 \mathrm{AM}]}\end{array}$ & $\begin{array}{c}t_{0, k, i}+[30-90] \mathrm{mins} \\
{[5: 00 \mathrm{PM} ; 7: 00 \mathrm{PM}]}\end{array}$ & $t_{f, k, i}+[0-30] \operatorname{mins}$ & $\begin{array}{l}{[0.15 ; 0.50]} \\
{[0.30 ; 0.55]}\end{array}$ & $\begin{array}{l}{[0.25 ; 0.80]} \\
{[0.75 ; 0.9]}\end{array}$ \\
\hline
\end{tabular}

(c) EV user synthesized behaviour - continued

to the ideal MMPC control from the EV user perspective. However, their optimal EV injections were different which explains why they incurred different grid constraint violations. This entails that the GULC controller smooths the charging process while still achieving close-to-ideal outcomes for EV users. Fig. 5c, however, shows that the GADC does not give the same guarantees. This is especially true for home chargers that on average provide EVs $10 \%$ and $20 \%$ less energy. This behaviour is in accordance to the probability violations, and lack there of in the case of GADC, shown in Figs. 3-4. It can be physically explained by two factors: (i) the high-loading conditions that happen at night-time when the simulated grid has no generating units, and (ii) the fact that in this simulation EV node placement and maximum number of connected EVs per node were chosen at random. This explains the sub-optimal performance of the GADC controller from the EV user's perspective. Indeed, the grid is simply not designed for such simulated high power unbalanced EV load demand. Therefore, as a final note, we believe that with proper CS placement planning together with the deployment of our proposed method (i.e. GADC controller), the network will remain safe and the EV user needs would be better fulfilled.

\section{CONCLUSION}

The paper proposed a grid-aware distributed control (GADC) for EV CSs connected to electrical grids of generic topologies. It uses ADMM to distribute a separably formulated problem into local ones, solved at the EV aggregators level, and a central grid aggregator problem that ensures the nonviolation of the grid operational and power quality constraints while taking into account all the solutions coming from the EV aggregators' problems. The scenario-based grid aggregator problem relies on a linearisation of the power-flow equations and is formulated for a generic three phase power grid. Therefore, it also takes into account voltage unbalances and guarantees proper power quality subject to the E50160 std. bounds. The performance of the method is illustrated on the IEEE-34 feeder by comparing it with two grid-unaware controllers. It is shown that the grid-unaware controllers may

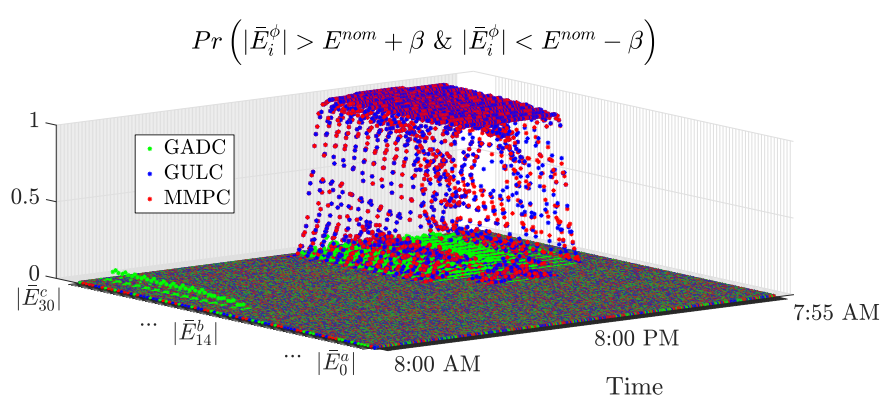

(a) Nodal voltage magnitudes

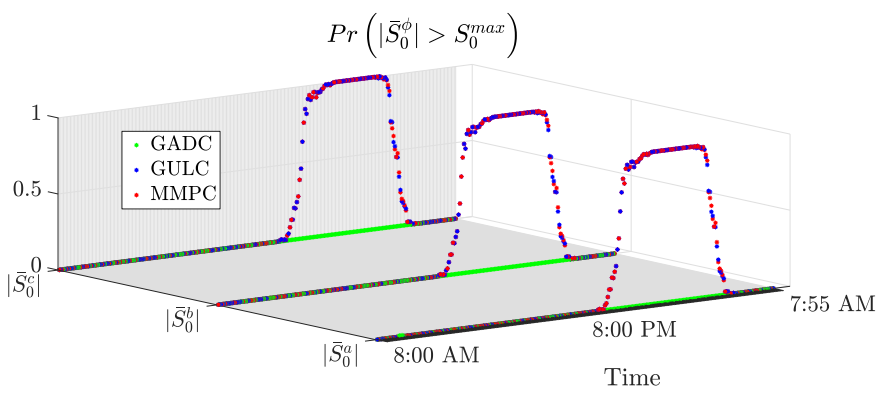

(b) Slack apparent power magnitudes

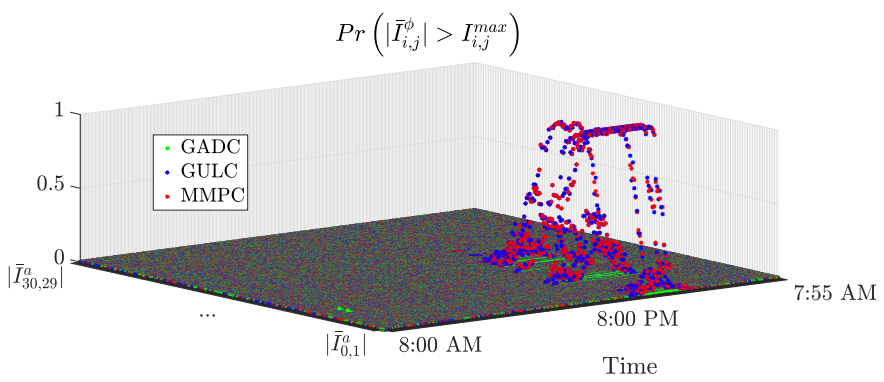

(c) Branch current magnitudes

Fig. 3. Post-LFMCS probabilities to violate grid operational constraints during the simulated 24 hours - $\mathrm{ABC}$ domain 


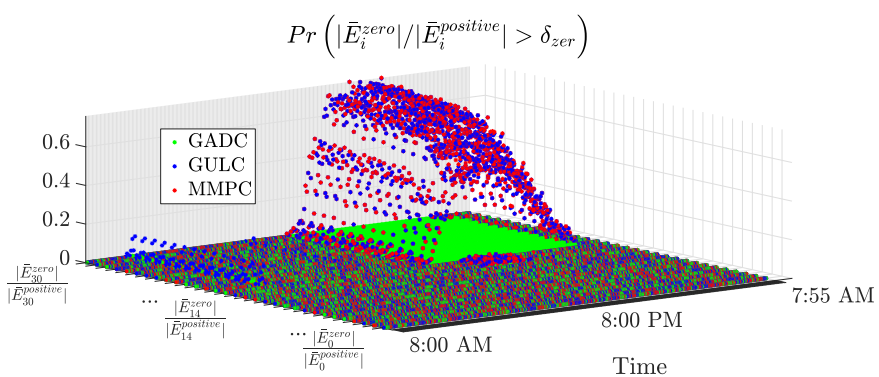

(a) Ratio of zero- and positive- sequence nodal voltage magnitudes

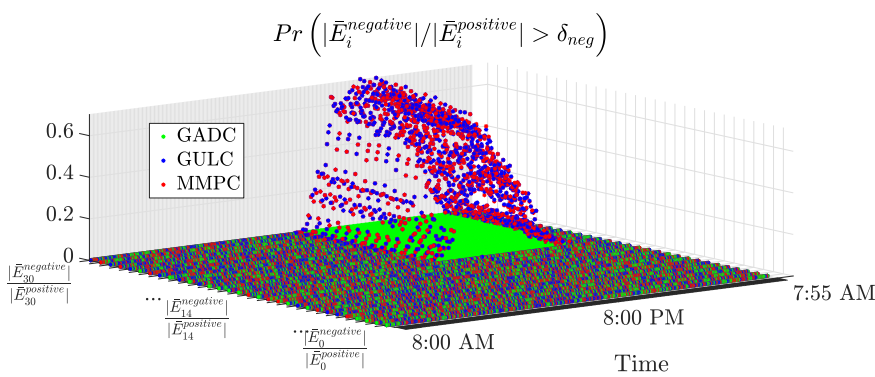

(b) Ratio of negative- and positive- sequence nodal voltage magnitudes

Fig. 4. Probabilities to violate grid constraints during the simulated 24 hours - sequence domain

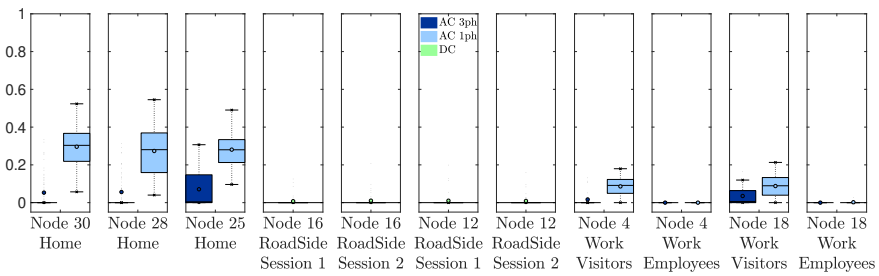

(a) MMPC

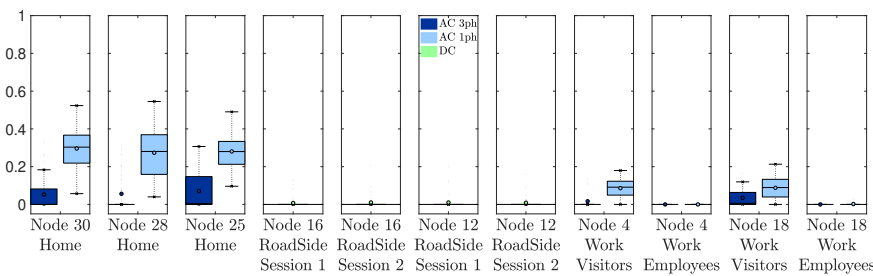

(b) GULC

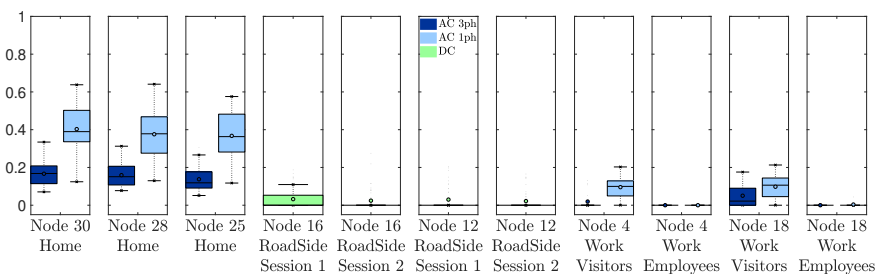

(c) GADC

Fig. 5. Difference between desired $\mathrm{SoC}$ at departure and actual one, for all nodes with CSs and for each simulated control algorithm provide a better service to $\mathrm{EV}$ users in terms of meeting desired battery energy levels at the expense of severely violating the grid security and power quality constraints. From the grid perspective the proposed GADC kept the power grid within the safe operational bounds and, in most cases, was also beneficial to EV users. It is clear that the developed method can help grid operators manage weak grids, that accommodate heavy EV loads, as best as they can. Future works plan to first, investigate the possibility of including model predictive control into the EV sub-problems, and, second, perform experimental validations of the GADC controller on a real-scale microgrid.

\section{REFERENCES}

[1] G. Putrus, P. Suwanapingkarl, D. Johnston, E. Bentley, and M. Narayana, "Impact of electric vehicles on power distribution networks," in 2009 IEEE Vehicle Power and Propulsion Conference. IEEE, 2009, pp. 827831

[2] Y. He, B. Venkatesh, and L. Guan, "Optimal scheduling for charging and discharging of electric vehicles," IEEE transactions on smart grid, vol. 3, no. 3, pp. 1095-1105, 2012.

[3] S. Deilami, A. S. Masoum, P. S. Moses, and M. A. Masoum, "Realtime coordination of plug-in electric vehicle charging in smart grids to minimize power losses and improve voltage profile," IEEE Transactions on Smart Grid, vol. 2, no. 3, pp. 456-467, 2011.

[4] P. Richardson, D. Flynn, and A. Keane, "Optimal charging of electric vehicles in low-voltage distribution systems," IEEE Transactions on Power Systems, vol. 27, no. 1, pp. 268-279, 2011.

[5] M. Liu, P. K. Phanivong, Y. Shi, and D. S. Callaway, "Decentralized charging control of electric vehicles in residential distribution networks," IEEE Transactions on Control Systems Technology, vol. 27, no. 1, pp 266-281, 2017.

[6] L. Zhang, V. Kekatos, and G. B. Giannakis, "Scalable electric vehicle charging protocols," IEEE Transactions on Power Systems, vol. 32, no. 2, pp. 1451-1462, 2016.

[7] K. Christakou, J.-Y. Le Boudec, M. Paolone, and D.-C. Tomozei, "Efficient computation of sensitivity coefficients of node voltages and line currents in unbalanced radial electrical distribution networks," IEEE Transactions on Smart Grid, vol. 4, no. 2, pp. 741-750, 2013.

[8] W. H. Kersting, "Radial distribution test feeders," in Power Engineering Society Winter Meeting, 2001. IEEE, vol. 2. IEEE, 2001, pp. 908-912.

[9] T. A. Short, Electric power distribution handbook. CRC press, 2014.

[10] J. Arrillaga and N. R. Watson, Power system harmonics. John Wiley \& Sons, 2004

[11] R. Gupta, F. Sossan, and M. Paolone, "Performance assessment of linearized opf-based distributed real-time predictive control," 2019.

[12] E. Dall'Anese, K. Baker, and T. Summers, "Chance-constrained ac optimal power flow for distribution systems with renewables," IEEE Transactions on Power Systems, vol. 32, no. 5, pp. 3427-3438, 2017.

[13] E. Standard, "50160," Voltage characteristics of public distribution systems, p. 18, 2010.

[14] F. Sossan, E. Namor, R. Cherkaoui, and M. Paolone, "Achieving the dispatchability of distribution feeders through prosumers data driven forecasting and model predictive control of electrochemical storage," IEEE Transactions on Sustainable Energy, vol. 7, no. 4, pp. 1762-1777, 2016.

[15] L. Yao, W. H. Lim, and T. S. Tsai, "A real-time charging scheme for demand response in electric vehicle parking station," IEEE Transactions on Smart Grid, vol. 8, no. 1, pp. 52-62, 2016.

[16] R. Gupta, F. Sossan, E. Scolari, E. Namor, L. Fabietti, C. Jones, and M. Paolone, "An admm-based coordination and control strategy for pv and storage to dispatch stochastic prosumers: Theory and experimental validation," in 2018 Power Syst. Comput. Conf., June 2018, pp. 1-7.

[17] S. Boyd, N. Parikh, E. Chu, B. Peleato, J. Eckstein et al., "Distributed optimization and statistical learning via the alternating direction method of multipliers," Foundations and Trends $\AA$ in Machine learning, vol. 3, no. 1, pp. 1-122, 2011.

[18] E. Stai, L. Reyes-Chamorro, F. Sossan, J.-Y. Le Boudec, and M. Paolone, "Dispatching stochastic heterogeneous resources accounting for grid and battery losses," IEEE Transactions on Smart Grid, vol. 9, no. 6, pp. 6522-6539, 2017. 